\title{
Prevalência de disfunção sexual masculina em adultos jovens
}

\section{Prevalence of male sexual dysfunction in young adults}

\author{
Natália Santos Pereira da Silva ${ }^{1 *}$, Silvana Maria de Macêdo Uchôa ${ }^{1}$, Valéria Conceição Passos \\ de Carvalho' ${ }^{1}$ Érica Patrícia Borba Lira Uchôa ${ }^{1}$
}

\begin{abstract}
RESUMO
O estudo buscou verificar a prevalência de disfunção sexual em homens adultos jovens e analisar possíveis correlações entre satisfação sexual e esquema corporal. Utilizou-se de cinco questionários em plataforma eletrônica Google Forms ${ }^{\circledR}$, em que, inicialmente, os participantes foram submetidos ao sócio-clínicodemográfico a fim de traçar o perfil da amostra e em seguida, foram aplicados o Índice Internacional da Função Erétil (IIEF-5), o Quociente Sexual - Versão Masculina (QS-M), o Inventário dos Esquemas de Gênero do Autoconceito (IEGA) e o Inventário de Satisfação Sexual de Golombok-Rust (GRISS). Obtevese uma amostra de 70 participantes, na qual, 74,3\% apresenta disfunção erétil leve, 4,3\% possui ejaculação precoce, destes, $18,6 \%$ se considera como insatisfeito sexualmente, contudo, $91,4 \%$ está satisfeito com o desempenho sexual. É possível concluir que a disfunção sexual masculina é multifatorial, a depender principalmente da pressão externa sofrida ou de patologias biopsicossociais. A disfunção erétil obteve maior prevalência na população estudada, o que impacta diretamente na satisfação sexual e qualidade de vida do indivíduo.
\end{abstract}

Palavras-chave: Sexualidade; Ereção peniana; Comportamento sexual; Disfunção erétil.

\begin{abstract}
The study sought to verify the prevalence of sexual dysfunction in young adult men and to analyze possible e correlations between sexual satisfaction and body schema. Five questionnaires were used on the electronic platform Google Forms ${ }^{\circledR}$, in which, initially, the participants were submitted to the socio-clinicaldemographic study in order to draw the profile of the sample and then, the International Index of Erectile Function (IIEF-5), the Sexual Quotient - Male Version (QS-M), the Self-Concept Gender Schemas Inventory (IEGA) and the Golombok-Rust Sexual Satisfaction Inventory (GRISS) were applied. A sample of 70 participants was obtained, in which $74.3 \%$ had mild erectile dysfunction, $4.3 \%$ had premature ejaculation, of these, $18.6 \%$ considered themselves sexually dissatisfied, however, $91.4 \%$ were satisfied with the sexual performance. It is possible to conclude that male sexual dysfunction is multifactorial, depending mainly on the external pressure suffered or biopsychosocial pathologies. Erectile dysfunction had a higher prevalence in the studied population, which directly impacts the individual's sexual satisfaction and quality of life.
\end{abstract}

Keywords: Sexuality; Penile erection; Sexual behavior; Erectile dysfunction.

\footnotetext{
${ }^{1}$ Universidade Católica de Pernambuco - UNICAP, Escola de Saúde e Ciências da Vida, Recife - Pernambuco, Brasil.

*E-mail: nsantosps2@gmail.com
} 


\section{INTRODUÇÃO}

Segundo a Organização Mundial da Saúde (OMS), 2014, a sexualidade "é uma energia que nos motiva para encontrar amor, contato, ternura e intimidade; ela integra-se no modo como sentimos, movemos, tocamos e somos tocados, é sentir-se sensual e ao mesmo tempo ser-se sexual. A sexualidade influencia pensamentos, sentimentos, ações e interações e, por isso, influencia também a nossa saúde física e mental". O conceito de sexualidade apenas como instinto já se encontra em desuso, apresentando sua importância significativa no que diz respeito aos aspectos sociais e psicológicos (SÁ; SANTOS, 2018). Além de agregar grande valor a qualidade de vida, é perceptível que se constitui uma característica inerente ao ser humano, ganhando-se um protagonismo único e indissociável, podendo variar de acordo com a estratificação social em que o individuo é exposto.

Toda e qualquer modificação neste vigor inato é conhecido como disfunção sexual, qualquer variação de uma das fases do ciclo de resposta sexual pode vir interligado a sintomatologia dolorosa, sentimento de frustração e constrangimento e diminuição considerável da prática sexual. (SOUZA et al, 2017; PINHEIRO; VINHOLES; TREVISOL,

Fatores biológicos, como genética, psicológicos, tal qual ansiedade e depressão, e sociais, a exemplo da falta de intimidade entre os parceiros, podem desencadear o transtorno sexual de maneira que cause perturbações psíquicas no sujeito que se encontra vivenciando esta situação. (LIDÓRIO, TATAREN, 2012)

Dentre as disfunções sexuais, as masculinas costumam passar despercebidas pelos profissionais de saúde e muito mais pelos homens expostos a essas desordens. Este fato deve-se a própria cultura hegemônica masculina que comumente tem maior dificuldade em procurar serviço médico, especialmente se tratando de uma causa de fórum íntimo (SERAFIM et al, 2019). A prevalência desses transtornos sexuais é bastante elevada, situando-se entre $31 \%$ a 46,2\%, (PINHEIRO; VINHOLES; TREVISOL, 2018). As formas mais predominantes de disfunção sexual são o transtorno do desejo sexual hipoativo, transtorno de aversão ao sexo, disfunção erétil, transtorno orgástico masculino, ejaculação precoce, baixa excitação sexual e dispareunia (LIDÓRIO, TATAREN, 2012). 
Estas disfunções podem ser potencializadas com a presença de ansiedade, devido à descarga de adrenalina no momento do ato sexual (BARBIERI FILHO, 2019).

As disfunções sexuais masculinas podem ser avaliadas de diversas formas e uma delas ocorre por meio de aplicação de questionários que, de modo geral, busca investigar a função sexual e erétil, ciclo de resposta sexual e satisfação pessoal com o coito. O Índice Internacional da Função Erétil é vastamente utilizado, principalmente na sua versão reduzida (IIEF-5), buscando a compreensão da função erétil masculina, assim como o Quociente Sexual - Versão Masculina, que além de avaliar a função propriamente dita, averigua as etapas do ciclo sexual e tudo que envolve o desempenho (GRAÇA 2008; PECHORRO, 2011). O Inventário dos Esquemas de Gênero do Autoconceito analisa a forma que o indivíduo se nota no meio em que vive, do mesmo modo que o Inventário de Satisfação Sexual Golombok-Rust, explora a relação entre parceiros e sua qualidade, interesse e frequência sexual (GIAVONI, 2000).

Diante do exposto, o presente estudo tem como finalidade avaliar a prevalência da disfunção sexual masculina na faixa etária entre 18 e 45 anos e compreender a influência das variáveis: satisfação sexual e autoesquema corporal na exacerbação do transtorno sexual, objetivando assim, maior conscientização e inserção do profissional da fisioterapia na área da andrologia que compreende a sexualidade como aspecto intrínseco da formação humana, própria da sua individualidade.

\section{MATERIAIS E MÉTODO}

Este projeto está vinculado a Universidade Católica De Pernambuco a Escola de Saúde e Ciências da Vida e ao curso de Fisioterapia. É parte integrante do projeto de pesquisa intitulado "ABORDAGENS FISIOTERAPÊUTICAS NAS DISFUNÇÕES SEXUAIS FEMININAS E MASCULINAS" sob coordenação de SILVANA MARIA DE MACEDO UCHÔA, cadastrado sob o $\mathrm{N}^{\circ}$ de CAAE 03239318.1.0000.5206, aprovado pelo comitê de ética e pesquisa em seres humanos, com o número do parecer 3.049.749 e pertencente ao grupo de Fisioterapia baseada em Evidências que será desenvolvido no Laboratório de Fisioterapia e Terapia Ocupacional Corpore Sano.

O estudo é de caráter descritivo-analítico, observacional, quantitativo e de corte transversal. A escolha da amostra foi feita de forma intencional, de acordo com o desejo dos indivíduos em participar da pesquisa. 
Foi estimada uma amostra inicial de 30 participantes, os quais foram informados do objetivo da pesquisa e a importância da sua participação, ressaltando que suas identidades seriam mantidas em sigilo. No entanto, com o decorrer da coleta de dados, observou-se que o número amostral ultrapassou a estimativa e totalizou 70 participantes, possibilitando uma melhor análise estatística.

Como critérios de inclusão adotou-se indivíduos do sexo masculino, ter idade entre 18 anos e 45 anos e ter vida sexual ativa. E como critérios de exclusão, indivíduos do sexo masculino que apresentem problemas neurológicos, cognitivos, doenças reumatológicas e afecções cardiovasculares severas que interfiram no sistema musculoesquelético.

Logo em seguida, era perguntado aos voluntários se eles aceitam participar do projeto, e caso concorde, foi solicitada a assinatura do Termo de Consentimento Livre Esclarecido - TCLE adaptado pelos pesquisadores. Somente após a assinatura deste termo era iniciada a coleta dos dados com a aplicação dos questionários.

Posteriormente, foi aplicado o questionário sócio-clínico-demográfico, formulado pelo avaliador, que consta dados pessoais como nome, idade, sexo, peso, altura, raça, escolaridade, estado civil e afiliação religiosa, a fim de traçar o perfil da amostra.

O estudo utilizou questionários que avaliam a condição sexual do indivíduo e a relação intima com o esquema corporal próprio correlacionando com a satisfação sexual dos envolvidos.

Inicialmente, foi aplicado o Índice Internacional da Função Erétil (IIEF), validado no Brasil em 2013 (GONZÁLES, 2013), designado de IIEF-5 como abreviação, tem por finalidade a avaliação da função erétil em homens. É um instrumento multidimensional e auto-administrável e possui uma alta sensibilidade para detectar mudanças na função erétil. Constituído por 5 questões, no qual cada item é pontuado em uma escala ordinal de 5 pontos, e com os valores mais baixos representando uma função sexual pior. O escore total do IIEF-5 varia de 5 a 25 pontos. O participante seleciona em cada questão uma das seis alternativas possíveis que melhor descreve sua situação (TABOSA, 2016).

Posteriormente, era utilizado o Quociente Sexual - Versão Masculina (QS-M), que é caracterizado por um questionário autoaplicável composto por 10 itens, constituído por 6 possíveis respostas que buscam avaliar a função sexual geral, etapas do ciclo de 
resposta sexual (desejo, excitação, orgasmo) e satisfação sexual. Neste questionário é possível avaliar as principais disfunções sexuais masculinas: transtorno do desejo sexual hipoativo, transtorno erétil e ejaculação precoce (LUCENA, 2018).

Em seguida, foi aplicado o Inventário dos Esquemas de Gênero do Autoconceito (IEGA), validada no Brasil em 2000, este questionário é baseado na percepção que o indivíduo possui sobre si mesmo. Compõe-se de 83 adjetivos que se relacionam com a personalidade do avaliado. Utilizando uma escala de cinco pontos, em que 0 é a ausência de identificação até 4, sugerindo total reconhecimento do item, é solicitado, então, que assinale o grau de identificação com cada item citado certificando total veracidade nas respostas escolhidas (GIOVANI; TAMAYO, 2000).

Por fim, foi aplicado o Inventário de Satisfação Sexual Golombok-Rust, GRISS, que abrange 28 questões que foram respondidas segundo uma escala tipo Likert que varia de 0 (nunca) a 4 (sempre) (SERAFIM et al, 2019). O inventário visou avaliar a forma em que os participantes lidam com seu próprio corpo e o do parceiro, o nível de interesse sexual, a qualidade da comunicação com o parceiro como também a frequência da prática sexual e a capacidade de atingir o clímax da relação, o orgasmo. (LUCAS, 2011).

Ao término da coleta de dados, os mesmos foram compilados em uma planilha Excel 2007® e feito uma análise descritiva (média, desvio padrão, mínimo e máximo). A análise estatística realizada utilizou os softwares Excel v16.31 e o SPSS v25.0, adotando como base um $\mathrm{p}<0,05$. A distribuição das variáveis mensuradas foi feita através de tabelas ou gráficos. Inicialmente foi aplicado o teste de Kolmogorov-Smirnov para testar a suposição de normalidade das variáveis envolvidas no estudo. Para verificar a existência de associação entre as escalas IIEF-5, QS-M, IEGA e GRISS foram calculados os coeficientes de correlação de Spearman. Já a fim de analisar as variáveis qualitativas segundo a classificação das escalas IIEF-5, QS-M e GRISS, foi aplicado o teste Quiquadrado ou exato de Fisher, quando necessário. Para a análise comparativa dos domínios do IEGA segundo o perfil sociodemográfico foi aplicado o teste não-paramétrico de Mann-Whitney ou de Kruskal-Wallis, com seus respectivos testes de comparações múltiplas (JERROLD, 1996). Todas as conclusões foram tomadas ao nível de significância de $5 \%$.

\section{RESULTADOS E DISCUSSÃO}


A amostra do presente estudo foi composta por 70 homens, sendo 22 deles com idades entre 19 a 43 anos e todos foram avaliados para identificar a existência de disfunção sexual.

A tabela 1 apresenta a distribuição dos adultos jovens avaliados quanto as características sociodemográficas. Nesta, é possível verificar que 58,6\% $(n=41)$ dos entrevistados tem idades de até 25 anos, $54,3 \%(n=38)$ tem altura de até $1,78 \mathrm{~m}, 48,6 \%$ $(\mathrm{n}=34)$ são de cor branca, $51,4 \%(\mathrm{n}=36)$ tem curso superior completo ou incompleto, 84,3\% (n=59) são solteiros, 85,7\% (n=60) são heterossexuais, 51,4\% (n=36) são agnósticos e 28,6\% (n=20) são estudantes.

Tabela 1 - Distribuição dos adultos jovens avaliados quanto as características sociodemográficas.

(continua)

\begin{tabular}{lll}
\hline $\begin{array}{l}\text { Características } \\
\text { sociodemográficas }\end{array}$ & $\mathrm{N}$ & $\%$
\end{tabular}

\section{Faixa etária}

Até 25

Altura

Até 1,78

Sexo

Masculino

\section{Raça/ cor}

Branca

Pardo

Mestiço

Negra

Preta

4

Morena

Sem informação 


\section{Escolaridade}

Ensino superior completo

Ensino superior incompleto

Ensino médio completo

Pós-graduação

Sem informação

\section{Estado Civil}

Solteiro

Casado

11

15,7

Tabela 1 - Distribuição dos adultos jovens avaliados quanto as características sociodemográficas.

(conclusão)

\begin{tabular}{lll}
\hline $\begin{array}{l}\text { Características } \\
\text { sociodemográficas }\end{array}$ & $\mathrm{N}$ & $\%$ \\
\hline
\end{tabular}

Orientação sexual

Heterossexual

60

85,7

Homossexual

5

7,1

Bissexual

2

2,9

Indefinido

3

4,3

\section{Religião}

Agnóstico

Católico

Evangélico

7

10,0

Espírita

Deísta

Umbandista

1

1,4

\section{Ocupação}

Estudante 


\begin{tabular}{lll} 
Autônomo/ Empresário & 6 & 8,6 \\
Administrador & 4 & 5,7 \\
Professor & 4 & 5,7 \\
Comerciante & 3 & 4,3 \\
Desempregado & 2 & 2,9 \\
Funcionário publico & 2 & 2,9 \\
Educador físico & 2 & 2,9 \\
Fisioterapeuta & 2 & 2,9 \\
Publicitário & 2 & 2,9 \\
Outros & 23 & 32,9 \\
\hline
\end{tabular}

A tabela 2 apresenta a distribuição dos adultos jovens avaliados quanto idade e altura. Nesta destacamos que a idade foi, em média, de 26,23 $\pm 5,0$ anos e a altura foi, em média, de $1,77 \pm 0,07$ metros.

Tabela 2 - Distribuição dos adultos jovens avaliados quanto idade e altura.

\begin{tabular}{lccccc}
\hline & $\mathbf{N}$ & Mínimo & Máximo & Média & Desvio \\
\hline Idade & 70 & 19,00 & 43,00 & 26,23 & 5,00 \\
Altura & 70 & 1,62 & 2,00 & 1,77 & 0,07 \\
\hline
\end{tabular}

A tabela 3 apresenta a distribuição dos jovens avaliados quanto a classificação dos instrumentos IIEF-5, QS-M e GRISS. Nesta destacamos que, em relação a avaliação da função erétil (IIEF-5), 74,3\% (n=52) dos entrevistados tem disfunção erétil leve. Verificamos ainda que nenhum apresenta transtorno do desejo sexual hipoativo, nenhum apresenta transtorno erétil e apenas 4,3\% (n=3) apresentam ejaculação precoce. Em relação a avaliação da função sexual, ciclo de resposta sexual e satisfação sexual, 91,4\% (n=64) estão satisfeitos com o desempenho sexual. E em relação, a avaliação da satisfação sexual (GRISS ${ }^{1}$ ) verificamos que 18,6\% ( $\left.\mathrm{n}=13\right)$ estão insatisfeitos. 
Tabela 3 - Distribuição dos jovens avaliados quanto a classificação dos instrumentos IIEF-5, QS-M e GRISS.

\begin{tabular}{lcc}
\hline Classificação instrumentos & $\mathbf{N}$ & $\%$ \\
\hline IIEF-5 & 10 & 14,3 \\
Sem DE & 52 & 74,3 \\
DE leve & 8 & 11,4 \\
DE leve a moderada &
\end{tabular}

\section{QS-M}

\section{Transtorno do desejo sexual hipoativo}

Com disfunção

0,0

Sem disfunção

\section{Transtorno erétil}

Com disfunção

$\begin{array}{cc}0 & 0,0 \\ 70 & 100,0\end{array}$

\section{Ejaculação precoce}

Com disfunção

Sem disfunção

\section{QS-M}

Bom a excelente

Regular a bom $35 \quad 50,0$

Desfavorável a regular

5

Ruim a desfavorável

1

\section{GRISS ${ }^{1}$}

Satisfeitos

1 Ponto de corte utilizado de 30.

A tabela 4 apresenta a distribuição dos adultos jovens avaliados quanto as características sociodemográficas segundo o QS-M. Nesta verificamos associação estatisticamente significante com a faixa etária. Assim podemos afirmar que avaliação da 
função sexual, ciclo de resposta sexual e satisfação sexual foi melhor entre os indivíduos com idade superior a 25 anos ( $\mathrm{p}$-valor $=0,053$ ).

Tabela 4 - Distribuição dos adultos jovens avaliados quanto as características sociodemográficas segundo o QS-M.

\begin{tabular}{|c|c|c|c|c|c|}
\hline \multirow{3}{*}{$\begin{array}{l}\text { Características } \\
\text { sociodemográficas }\end{array}$} & \multicolumn{4}{|c|}{ QS-M } & \multirow{3}{*}{$\begin{array}{c}\text { p- } \\
\text { valor }\end{array}$} \\
\hline & $\begin{array}{c}\text { Bom a } \\
\text { excelente }\end{array}$ & $\begin{array}{l}\text { Regular a } \\
\text { bom }\end{array}$ & $\begin{array}{c}\text { Desfavorá } \\
\text { vel a } \\
\text { regular }\end{array}$ & $\begin{array}{c}\text { Ruim a } \\
\text { desfavorá } \\
\text { vel }\end{array}$ & \\
\hline & $\%$ & $\mathbf{N}$ & $\mathbf{N}$ & $\mathbf{N}$ & \\
\hline
\end{tabular}

\section{Faixa etária}

$\begin{array}{llllllllll}\text { Até } 25 & 12 & 29,3 & 25 & 61,0 & 3 & 7,3 & 1 & 2,4 & \\ >25 & 17 & 58,6 & 10 & 34,5 & 2 & 6,9 & 0 & 0,0 & \mathbf{0 , 0 5 3}\end{array}$

Altura

Até 1,78

$\begin{array}{llllllll}18 & 47,4 & 17 & 44,7 & 2 & 5,3 & 1 & 2,6\end{array}$

$>1,78$

$\begin{array}{lllllllll}11 & 34,4 & 18 & 56,3 & 3 & 9,4 & 0 & 0,0 & 0,511\end{array}$

Raça/ cor

$\begin{array}{lccccccccc}\text { Branca } & 14 & 41,2 & 18 & 52,9 & 1 & 2,9 & 1 & 2,9 & \\ \text { Parda } & 9 & 42,9 & 8 & 38,1 & 4 & 19,0 & 0 & 0,0 & \\ \text { Outras } & 5 & 35,7 & 9 & 64,3 & 0 & 0,0 & 0 & 0,0 & 0,267\end{array}$

Escolaridade

$\begin{array}{lccccccccc}\text { Ensino superior } & 16 & 35,6 & 26 & 57,8 & 3 & 6,7 & 0 & 0,0 & \\ \text { Ensino médio } & 11 & 47,8 & 9 & 39,1 & 2 & 8,7 & 1 & 4,3 & \\ \text { Pós-graduação } & 2 & 100,0 & 0 & 0,0 & 0 & 0,0 & 0 & 0,0 & 0,246\end{array}$

\section{Estado Civil}

$\begin{array}{lccccccccc}\text { Solteiro } & 22 & 37,3 & 32 & 54,2 & 4 & 6,8 & 1 & 1,7 & \\ \text { Casado } & 7 & 63,6 & 3 & 27,3 & 1 & 9,1 & 0 & 0,0 & 0,304\end{array}$




\section{Orientação sexual}

$\begin{array}{lccccccccc}\text { Heterossexual } & 23 & 38,3 & 31 & 51,7 & 5 & 8,3 & 1 & 1,7 & \\ \text { Outras } & 6 & 60,0 & 4 & 40,0 & 0 & 0,0 & 0 & 0,0 & 0,555\end{array}$

\section{Religião}

\begin{tabular}{lccccccccc} 
Agnóstico & 12 & 33,3 & 19 & 52,8 & 4 & 11,1 & 1 & 2,8 & \\
Católico & 12 & 52,2 & 10 & 43,5 & 1 & 4,3 & 0 & 0,0 & \\
Outras & 5 & 45,5 & 6 & 54,5 & 0 & 0,0 & 0 & 0,0 & 0,733 \\
\hline
\end{tabular}

A tabela 5 apresenta a distribuição dos adultos jovens avaliados quanto as características sociodemográficas segundo o fator de racionalidade. Nesta verificamos associação significativa com a orientação sexual. Assim podemos afirmar que o escore de racionalidade foi maior entre os indivíduos que não são heterossexuais (pvalor $=0,016$ ).

Tabela 5 - Distribuição dos adultos jovens avaliados quanto as características sociodemográficas segundo o fator de racionalidade.

\begin{tabular}{lcccccc}
\hline Fator racionalidade & N & $\begin{array}{c}\text { Míni } \\
\text { mo }\end{array}$ & $\begin{array}{c}\text { Máxi } \\
\text { mo }\end{array}$ & Média & Desvio & $\begin{array}{c}\text { p- } \\
\text { valor }\end{array}$ \\
\hline Faixa etária & & & & & & \\
Até 25 & 41 & 0,38 & 3,00 & 1,70 & 0,59 & \\
$>25$ & 29 & 0,38 & 2,88 & 1,81 & 0,67 & 0,259
\end{tabular}

\section{Altura}

Até 1,78

$38 \quad 0,38 \quad 2,50 \quad 1,68 \quad 0,61$

$>1,78$

$32 \quad 0,38 \quad 3,00 \quad 1,83 \quad 0,64 \quad 0,408$

\section{Raça/ cor}

$\begin{array}{lllllll}\text { Branca } & 34 & 0,75 & 2,75 & 1,74 & 0,51 & \\ \text { Parda } & 21 & 0,38 & 3,00 & 1,78 & 0,74 & \\ \text { Outras } & 14 & 0,38 & 2,88 & 1,67 & 0,72 & 0,803\end{array}$




\section{Escolaridade}

Ensino superior

$45 \quad 0,38 \quad 3,00 \quad 1,76 \quad 0,65$

Ensino médio

$\begin{array}{lllll}23 & 0,75 & 2,75 & 1,71 & 0,58\end{array}$

Pós-graduação

2

$\begin{array}{lllll}1,25 & 2,38 & 1,81 & 0,80 & 0,897\end{array}$

\section{Estado Civil}

Solteiro

$\begin{array}{lllll}59 & 0,38 & 3,00 & 1,69 & 0,63\end{array}$

Casado

$\begin{array}{llllll}11 & 0,88 & 2,88 & 2,02 & 0,55 & 0,085\end{array}$

\section{Orientação sexual}

Heterossexual

Outras
60

0,38

2,88

1,68

10

1,13

3,00

2,16

0,62

0,53

0,016

\section{Religião}

Agnóstico 36

0,38

2,75

1,68

0,68

Católico

23

0,88

2,50

$1,86 \quad 0,46$

Outras

$11 \quad 0,88 \quad 3,00$

1,74

0,77

0,577

A tabela 6 apresenta a distribuição dos jovens avaliados quanto as características sociodemográficas segundo o fator de ousadia. Nesta verificamos associação significativa com a orientação sexual. Assim podemos afirmar que o escore de ousadia foi maior entre os indivíduos que não são heterossexuais ( $\mathrm{p}$-valor=0,007).

Tabela 6 - Distribuição dos jovens avaliados quanto as características sociodemográficas segundo o fator de ousadia.

\begin{tabular}{lccccccc}
\hline Fator ousadia & N & $\begin{array}{c}\text { Mínim } \\
\text { o }\end{array}$ & $\begin{array}{c}\text { Máxi } \\
\text { mo }\end{array}$ & Média & Desvio & $\begin{array}{c}\text { p- } \\
\text { valor }\end{array}$ \\
\hline Faixa etária & & & & & & \\
Até 25 & 41 & 0,20 & 2,80 & 1,92 & 0,68 & \\
$>25$ & 29 & 0,60 & 3,40 & 2,08 & 0,70 & 0,483
\end{tabular}


Altura

Até 1,78

$38 \quad 0,20 \quad 3,20 \quad 1,90 \quad 0,71$

$>1,78$

3,40

2,09

0,65

0,169

\section{Raça/ cor}

Branca

$34 \quad 0,80 \quad 3,20 \quad 2,00 \quad 0,59$

Parda

$\begin{array}{lllll}21 & 0,60 & 3,00 & 2,00 & 0,74\end{array}$

Outras

$14 \quad 0,20 \quad 3,40 \quad 1,93 \quad 0,89 \quad 0,941$

\section{Escolaridade}

Ensino superior

$45 \quad 0,20 \quad 3,40 \quad 2,01 \quad 0,71$

Ensino médio

$23 \quad 0,80 \quad 2,80 \quad 1,93 \quad 0,66$

Pós-graduação

$2 \quad 1,60$

2,80

2,20

$0,85 \quad 0,898$

\section{Estado Civil}

Solteiro

$59 \quad 0,20 \quad 3,20 \quad 1,95 \quad 0,69$

Casado

$11 \quad 1,00 \quad 3,40 \quad 2,20 \quad 0,67 \quad 0,446$

\section{Orientação sexual}

Heterossexual

$60 \quad 0,20 \quad 3,40 \quad 1,90 \quad 0,68$

Outras

$10 \quad 1,40 \quad 3,20 \quad 2,50 \quad 0,55$

$\mathbf{0 , 0 0 7}$

\section{Religião}

$\begin{array}{lllllll}\text { Agnóstico } & 36 & 0,20 & 3,20 & 1,89 & 0,75 & \\ \text { Católico } & 23 & 1,00 & 3,00 & 2,16 & 0,47 & \\ \text { Outras } & 11 & 1,00 & 3,40 & 1,95 & 0,85 & 0,319\end{array}$

A disfunção sexual masculina pode ser considerada um problema de saúde pública e por muitas vezes, por não ser explorada, os enfermos convivem com esta desordem por anos e anos. (Kern, 2010). É perceptível que há grande dificuldade em retratar intimamente sobre sexualidade. Enquanto sociedade, os homens sofrem pressões externas como se necessitassem estar sempre prontos para o ato sexual e não pudessem nunca 
falhar ou negar. A partir desse receio, de frustação ou fracasso, podem vir a desenvolver esta condição (SILVEIRA, 2018). Na pesquisa atual, foi possível perceber o temor dos participantes ao retratarem sua condição sexual com devida veracidade mesmo que tenha sido assegurado que os dados obtidos seriam mantidos em sigilo e serviriam apenas para a conclusão do presente estudo.

A média de idade dos participantes do presente estudo foi de $26,23 \pm 5,0$ anos de idade, dado este que apresentou certa similaridade com os estudos de BRITTO (2010), o qual apresentou a sua idade média de 31,21 $\pm 6,16$ anos. Sugere-se, a partir disso, que a incidência aumente com o passar da idade e que o início dos sintomas das possíveis disfunções sexuais masculina seja comum entre o final da segunda década de vida e início da terceira década de vida (McCABE, 2016). De acordo com a escolaridade e o estado civil dos participantes, foi possível observar que $51,4 \%$ da população estudada possuíam ensino superior, completo ou incompleto, e 84,3\% eram solteiros, demostrando correlação com o estudo de SILVEIRA (2018), no qual, entre os estados de São Paulo, Rio Grande do Sul e Bahia, a população paulista analisada possuía maiores taxas de educação, sendo $96,5 \%$ de ensino fundamental e $68,8 \%$ do ensino médio, presume-se com isso, que os indivíduos deem continuidade a sua vida acadêmica, atingindo por conseguinte o ensino superior. A respeito do estado civil, os paulistanos adquiriram o maior indicativo de solteiros dentre os estudados, 9\%, ratificando a coerência com o estudo em questão.

A prática terapêutica conta com diversas formas de avaliação, seja ela por meio de exames físicos ou o emprego de escalas especificas para que possa direcionar o diagnóstico mais preciso. Com o Índice Internacional da Função Erétil, IIEF-5, foi possível classificar que $74,3 \%$ da amostra apresenta disfunção erétil leve, confirmado pelo estudo de YIKILMAZ (2019), que dentre os 68 homens avaliados, 31 apresentaram a mesma patologia, possibilitando assim levantar a hipótese de a disfunção erétil ser a disfunção sexual mais prevalente entre os homens. Também conhecida como impotência sexual, refere-se à incapacidade de sustentar ou alcançar a ereção até a finalização do ato sexual. De acordo com o Quociente Sexual - versão Masculina, que contém itens de avaliação física e emocional voltados para função sexual, apenas 4,3\% manifestam ejaculação precoce e de modo geral, a função sexual era considerada de regular a boa (50\%), que de maneira implícita, supõe-se contentamento com a satisfação sexual. No estudo de COUTINHO (2017), os achados demonstram que, entre os 26 homens 
submetidos, 8 deles (30,8\%), apresentavam desempenho sexual nulo a ruim, discordando do presente estudo.

O Inventário de Satisfação Sexual de Golombok-Rust, GRISS, é caracterizado pelo julgamento do funcionamento sexual, neste caso, masculino. Identifica-se que um percentual pequeno da população estudada $(18,6 \%)$ se enquadra como insatisfeitos sexualmente, validado pelos estudos de SERAFIM (2019) e GALATI (2014). Apresentando relação com lazer, a diminuição da prática traz consigo grande correlação com a satisfação sexual. O momento de descontração promove no corpo humano a liberação de opioides endógenos, como serotonina e ocitocina, considerados como hormônios do prazer, de suma importância para sobrevivência humana e no ato sexual, há um desprendimento demasiado da ocitocina, propiciando vínculo e alegria entre os parceiros.

No estudo em questão, foi evidenciado que há correlação entre o QS-M e a idade do indivíduo. Apesar de não ter sido encontrado artigos que comprovem tal convergência, imagina-se que à medida que o homem amadurece, o conhecimento íntimo se amplia. A partir deste dado momento, é possível perceber seus gostos e preferências no ato, aprimorando sua função sexual, juntamente com as etapas do ciclo que se compõe como desejo, excitação e orgasmo propriamente dito, repercutindo positivamente na satisfação sexual.

O Inventário dos Esquemas de Gênero do Autoconceito, IEGA, é voltada para avaliação da leitura que o indivíduo tem de si e para que essa análise seja feita, são priorizados fatores. O fator racionalidade refere-se a comportamentos racionais com distanciamento emocional, já o fator ousadia, refere-se à capacidade de adaptação e busca do próprio prazer. Obteve-se que, nas duas variáveis, há uma relação estatisticamente significativa com aqueles que não são heterossexuais. Segundo TEIXEIRA e CARDOSO (2017), a maior insatisfação a respeito da imagem corporal do homem homossexual relaciona-se com o receio de transmitir uma informação de mera feminilidade, diminuindo assim o interesse amoroso por parte de outros homens que venham a preferir masculinidade, dessa forma, discordando da presente pesquisa. Por outro lado, SILVEIRA e SANTOS (2019), afirmam que a abertura de discorrer sobre sua orientação sexual e vivenciá-la plenamente está diretamente ligada a satisfação sexual, sendo reduzido o peso do processo de aceitação e experenciar a liberdade de amar. 


\section{CONCLUSÃO}

Deste modo, é possível concluir que a disfunção sexual masculina é multifatorial, a depender principalmente da pressão externa sofrida ou de patologias biopsicossociais. Dentre elas, a disfunção erétil obteve maior prevalência na população estudada, o que impacta diretamente na satisfação sexual e qualidade de vida do indivíduo. São necessárias maiores pesquisas na área da andrologia a fim de que se obtenha dados mais consistentes a respeito destes distúrbios com o objetivo de traçar o tratamento fisioterapêutico mais apropriado e compatível.

\section{REFERÊNCIAS}

BARBIERI FILHO, A. Abordagem biopsicossocial de um caso de disfunção sexual masculina. Revista Brasileira de Sexualidade Humana, Ribeirão Preto, v. 30, n. 1, p. 80-84, jun. 2019.,

BRITTO, R.; BENETTI, S. P. C. Ansiedade, depressão e característica de personalidade em homens com disfunção sexual. Revista Sociedade Brasileira de Psicologia Hospitalar. Rio de Janeiro, v. 13, n. 2, p. 243-258, dez. 2010.

COUTINHO, P. J. R. Satisfação sexual de homens prostatectomizados após tratamento da disfunção erétil. 2017. 33 f. Tese (Mestrado) - Curso de Psicologia e Saúde, Faculdade de Medicina de São José do Rio Preto, São José do Rio Preto, 2017.

GALATI, M. C. R. et al. Sexualidade e qualidade de vida em homens com dificuldades sexuais. Psico-USF, Bragança Paulista, v. 19, n. 2, p. 243-252, ago. 2014.

GIAVONI, A.; TAMAYO, Á. Inventário dos Esquemas de Gênero do Autoconceito (IEGA). Psicologia: Teoria e Pesquisa, Brasília, v. 16, n. 2, p. 175-184, Agosto, 2000.

GONZÁLES, A. I. et al.; Validação do Índice Internacional de Função Erétil (IIFE) para uso no Brasil. Florianópolis, SC - Brasil, Novembro, 2012

JERROLD, H. Zar. Biostatistical analysis. Third edition, 1996. Prentice Hall, New Jersey.

KERN, C. A. R. Disfunção sexual masculina: compreensão psicanalítica. 2010. 120 f. Tese (Mestrado) - Curso de Psicologia, Universidade do Vale do Rio dos Sinos, São Leopoldo, 2010.

LIDÓRIO, A. A.; TATAREN, J. C. Disfunções sexuais masculinas. Universidade Estadual de Londrina, Centro de Ciências Biológicas, Departamento de psicologia geral e análise do comportamento, Psicologia clínica na análise do comportamento, out, 2012.

LUCAS, C. O. Ciúme e Satisfação Sexual na População Portuguesa. 2011. 139 f. Dissertação (Mestrado) - Curso de Psicologia, Universidade da Beira Interior, Covilhã, 2011. 
LUCENA, B. B. Fatores cognitivos na função sexual: adaptação transcultural e estudo psicométrico de instrumentos de medida em sexualidade. 2018. $124 \mathrm{f}$. Tese (Doutorado) - Curso de Psiquiatria, Faculdade de Medicina da Universidade de São Paulo, São Paulo, 2018.

McCABE, M. P. et al. Incidence and prevalence of sexual dysfunction in women and men: a consensus statement from the fourth international consultation on sexual medicine 2015. The Journal of Sexual Medicine. Melboourne, Australia. v.13, p. 144-152. 2016.

PINHEIRO, F. K. B.; VINHOLES, D. B.; TREVISOL, F. S. Prevalência de disfunção sexual e fatores associados entre policiais militares. Revista Brasileira de Medicina, Santa Catarina, p. 1-5, out. 2018.

SÁ, A. A. M.; SANTOS, C. V. M. A Vivência da Sexualidade de Pessoas que Vivem com HIV/Aids. Psicologia: Ciências e Profissão, Goiânia, v. 38, n. 4, p. 773-786, Out/Dez, 2018.

SERAFIM, A. F. V. et al. Avaliação da satisfação sexual de homens atendidos em ambulatório de urologia. Revista Eletrônica Acervo Saúde, Goiás, v. 11, n. 4, p. 1-9, jan. 2019.

SILVEIRA, A. P.; CERQUEIRA-SANTOS, E. Homofobia internalizada e satisfação sexual em casais homossexuais. Revista Psicogente. São Cristóvão, Sergipe. v. 22, n. 41, p. 1-18, jan. 2019.

SILVEIRA, D. G. et al. Educação sexual: perfil sociodemográfico de homens em disfunções sexuais e processo de ruptura da personificação da masculinidade. $3^{\circ}$ Encontro de ciências em educação para sustentabilidade. Universidade Luterana do Brasil, Canoas, Rio Grande do Sul, Nov. 2018.

SOUZA, F. H. C. et al. Análise da função sexual de pacientes com dermatomiosite e polimiosite através de questionários autoaplicados: um estudo transversal. Revista Brasileira de Reumatologia, São Paulo, v. 57, n. 2, p. 134-140, janeiro, 2017.

TABOSA, A. R. S. Disfunção erétil como preditor de pior qualidade de vida nos pacientes com doença arterial coronariana. 2016. 135 f. Tese Mestrado - Curso de Ciências da Saúde, Universidade Federal de Pernambuco, Recife, 2016.

TEIXEIRA, F. A.; CARDOSO, F. L. Orientação sexual e fatores associados em homens homossexuais. Revista do Departamento de Educação Física e Saúde. Florianópolis, Santa Catarina. v. 18, n. 2, p. 88-92, jun. 2017.

YIKILMAZ, T. N. et al. Evaluation of sexual dysfunction prevalence in infertile men with non-obstructive azoospermia. Archivio Italiano di Urologia e Andrologia. Ankara, Turquia. v. 91, n. 4, p. 241-244. 2019.

Recebido em: 15/10/2021

Aprovado em: 10/11/2021

Publicado em: 12/11/2021 